\title{
Normal and Inverse Diffusive Isotope Fractionation of Deuterated Toluene and Benzene in Aqueous Systems
}

\author{
Rolle, Massimo; Jin, Biao
}

Published in:

Environmental Science \& Technology Letters

Link to article, DOI:

10.1021/acs.estlett.7b00159

Publication date:

2017

Document Version

Peer reviewed version

Link back to DTU Orbit

Citation (APA):

Rolle, M., \& Jin, B. (2017). Normal and Inverse Diffusive Isotope Fractionation of Deuterated Toluene and Benzene in Aqueous Systems. Environmental Science \& Technology Letters, 4(7), 298-304.

https://doi.org/10.1021/acs.estlett.7b00159

\section{General rights}

Copyright and moral rights for the publications made accessible in the public portal are retained by the authors and/or other copyright owners and it is a condition of accessing publications that users recognise and abide by the legal requirements associated with these rights.

- Users may download and print one copy of any publication from the public portal for the purpose of private study or research.

- You may not further distribute the material or use it for any profit-making activity or commercial gain

- You may freely distribute the URL identifying the publication in the public portal 
This is a Post Print of the article published on line $31^{\text {st }}$ May 2017 in Environmental Science \& Technology Letters. The publishers' version is available at the permanent link: doi: $\underline{10.1021 / \text { acs.estlett.7b00159 }}$

\title{
Normal and Inverse Diffusive Isotope Fractionation of Deuterated Toluene and Benzene in Aqueous Systems
}

\author{
Massimo Rolle ${ }^{*, 1}$ and Biao Jin ${ }^{1}$
}

${ }^{1}$ Department of Environmental Engineering, Technical University of Denmark, Bygningstorvet Building 115, DK-2800 Kgs. Lyngby, Denmark

Corresponding author phone: +45 45251566; e-mail: masro@env.dtu.dk

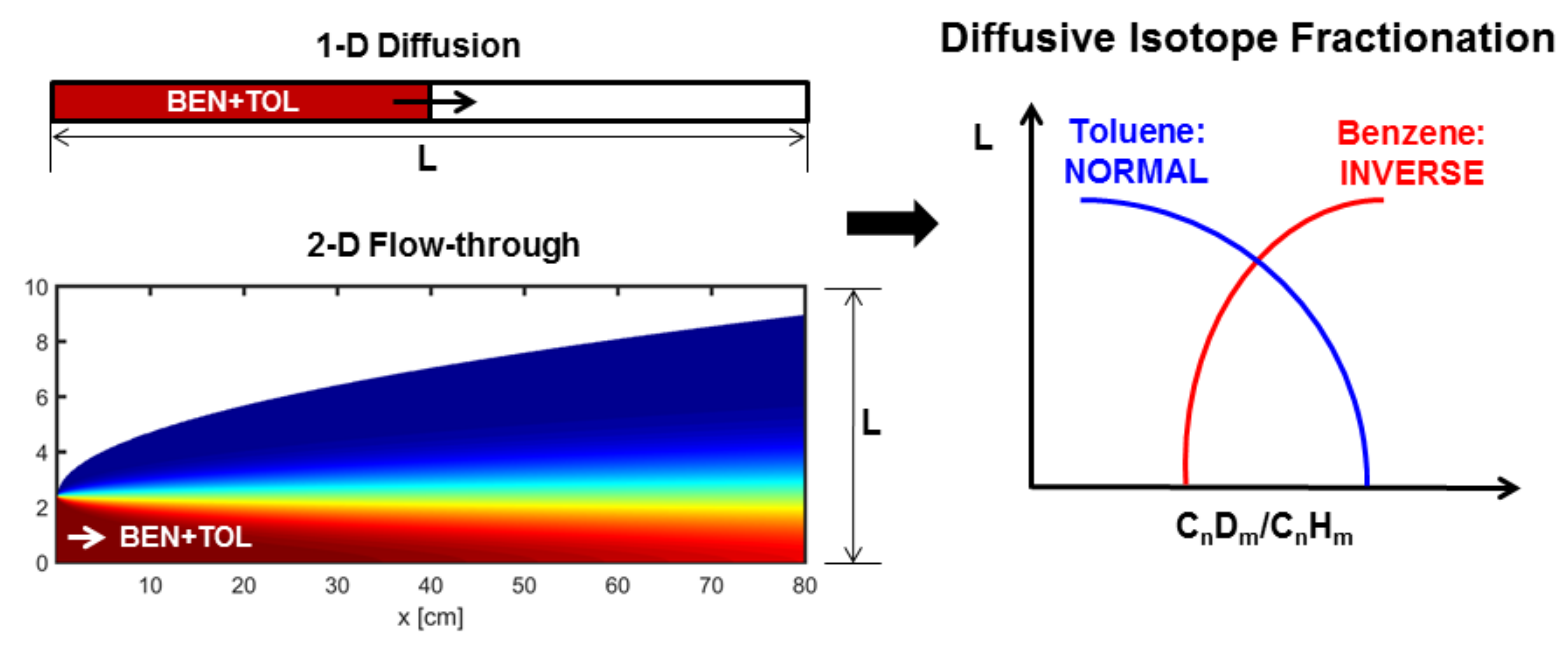




\section{Abstract}

2 Diffusive isotope fractionation of organic contaminants in aqueous solution is difficult to quantify

3 and only a few experimental datasets are available for compounds of environmental interest. In this

4 study, we investigate diffusive fractionation of perdeuterated and non-deuterated benzene and

5 toluene. Multitracer experiments were carried out in 1-D gel dissection tubes and in a quasi 2-D

6 flow-through porous medium. The experiments allowed us to simultaneously and directly compare

7 the diffusive and dispersive behavior of benzene and toluene. We observed an unexpected, opposite

8 behavior of the two monoaromatic hydrocarbons. Toluene showed a normal diffusive isotope effect

$9 \quad\left(\mathrm{D}_{\mathrm{C} 7 \mathrm{D} 8} / \mathrm{D}_{\mathrm{C} 7 \mathrm{H} 8}=0.96\right)$ with enrichment of the non-deuterated isotopologue in the direction of the 10 diffusive and transverse dispersive fluxes. Conversely, the measured trends for benzene indicate 11 inverse diffusive fractionation $\left(\mathrm{D}_{\mathrm{C} 6 \mathrm{D} 6} / \mathrm{D}_{\mathrm{C} 6 \mathrm{H} 6}=1.02\right)$, with a remarkably faster diffusion rate of the 12 perdeuterated isotopologue that was enriched in the downgradient portion of the diffusion tubes and 13 at the fringes of the contaminant plumes in the flow-through setup. These outcomes can neither be 14 interpreted as mass-dependent fractionation nor be described as purely hydrodynamic (i.e., mass 15 independent) effects. The results of this study are relevant for the use of labeled/non-labeled 16 mixtures of organic compounds as conservative and (bio)reactive tracers in environmental 17 applications. 


\section{Introduction}

Aromatic hydrocarbons are widespread contaminants, frequently found in soils and aquatic environments. Among these chemicals BTEX (i.e., benzene, toluene, ethylbenzene and xylene) compounds are of particular concern due to their relatively high solubility, mobility and toxicity. ${ }^{1}$ Mixtures of labeled and nonlabeled compounds have been often applied as a diagnostic tool to understand and quantify contaminant transport and transformation mechanisms. For instance, in the field of groundwater contamination, deuterium-labeled BTEX compounds have been used in tracer tests, push-pull tests and in situ microcosms to evaluate contaminant retardation and in situ rates of transformation during groundwater bioremediation and natural attenuation. ${ }^{2-6}$ One important aspect that has not (yet) been evaluated in detail is the extent of diffusive isotope fractionation of BTEX compounds in aqueous solution and its implications in environmental systems. The evaluation of such effect is important since recent investigation of subsurface solute transport has highlighted the key controlling role of aqueous diffusion for groundwater contaminant transport at different scales. ${ }^{7-10}$ Despite the increased recognition of the quantitative importance and of the macroscopic impact of small scale diffusive processes on large scale transport of organic contaminants, only a few experimental and modeling studies have attempted to quantify diffusive isotope fractionation for organic compounds. ${ }^{11-16}$ The lack of data and mechanistic understanding of organic chemicals' diffusive isotope fractionation becomes apparent when compared with the advances in the related field of inorganic isotope geochemistry, in which numerous studies have been carried out to investigate diffusive isotope effects of major cations, anions and dissolved gases in both aqueous solutions ${ }^{17-29}$ and non-aqueous systems. ${ }^{30-33}$

In this work we focus on diffusive transport of perdeuterated and non-deuterated benzene and toluene. The diffusive behavior of these chemicals (particularly of benzene) was investigated in early studies, ${ }^{34-36}$ mostly as self-diffusion or as tracer diffusion in organic solvents. However, to the 
best of our knowledge, no study has reported a comparison of diffusive isotope fractionation of these contaminants in aqueous solution. The purpose of this Letter is to report the unexpected, contrasting fractionation behavior of toluene and benzene that we have observed in a series of experiments performed in different setups under purely diffusive conditions (1-D gel dissection tubes), as well as in flow-through systems (2-D flow-through chamber). A key feature of the experiments was the simultaneous presence of non-deuterated and perdeuterated mixtures of toluene and benzene undergoing diffusion and lateral dispersion. In this way, in each experiment the 4 tracers $\left(\mathrm{C}_{6} \mathrm{H}_{6}, \mathrm{C}_{6} \mathrm{D}_{6}, \mathrm{C}_{7} \mathrm{H}_{8}, \mathrm{C}_{7} \mathrm{D}_{8}\right)$ were all transported under the same conditions, which facilitates a direct comparison of the effects of diffusion and transverse dispersion on the concentration of non-deuterated and perdeuterated benzene and toluene and, more importantly, on their diffusive isotope fractionation.

\section{Materials and Methods}

Chemicals and Analytical Methods. High-purity organic compounds (99.5\%, Sigma-Aldrich, Germany) were used in the experiments. Measurements were carried out with a 7890A gas chromatograph (GC) with a capillary column $(30 \mathrm{~m} \times 250 \mu \mathrm{m}, 1.0 \mu \mathrm{m}$ film thickness; Agilent, USA) coupled to a 5975C tri-axis mass selective detector (MSD) (Agilent, USA). Headspace samples were injected for analysis using a COMBIPAL multi-purpose autosampler system.

1-D Diffusion Experiment. Gel dissection experiments were performed in cylindrical glass tubes (1.1 $\mathrm{cm}$ diameter and $20 \mathrm{~cm}$ length) using agarose gel prepared with a minimum amount $(1 \% \mathrm{w} / \mathrm{w})$ of phyto agar (Duchefa, Netherlands). Agar solutions containing mixtures of dissolved toluene, perdeuterated toluene, benzene and perdeuterated benzene (1:1:1:1 volume proportion) were prepared and filled in the first $10 \mathrm{~cm}$ of the diffusion tubes. This zone acted as contaminant source during the experiments in which the compounds diffused towards the remaining portion of the tubes 
that was filled with pure gel medium. The tubes were kept horizontally at a constant temperature of $20{ }^{\circ} \mathrm{C}$. After 9 and 15 days, the tubes were sampled by cutting the gel into $1 \mathrm{~cm}$ slices with a scalpel. The slices were immediately sealed in $10 \mathrm{ml}$ glass vials with screw caps and sent to GC analysis.

2-D Flow-through Experiment. Flow-through experiments were performed in a quasi twodimensional flow-through chamber (inner dimensions: $80 \mathrm{~cm} \times 18 \mathrm{~cm} \times 1 \mathrm{~cm}, \mathrm{~L} \times \mathrm{H} \times \mathrm{W}$ ), equipped with 10 equally-spaced $(1 \mathrm{~cm}$ spacing) ports both at the inlet and at the outlet. The flow-through system was filled with homogeneous quartz sand (Euroquarz, Germany) with grain diameter of 1.0-1.5 mm. The sand was washed in an acidic solution and dried for 12 hours in an oven at $120^{\circ} \mathrm{C}$ before filling the flow-through chamber. The sand was filled with a wet-packing procedure using ultra-pure MilliQ water (EvoquaWater, USA) to avoid air entrapment in the porous medium. ${ }^{37}$ The inlet and outlet ports were connected to two high-precision multi-channel peristaltic pumps (IPC-N24, Ismatec, Switzerland). Sampling was performed with a 10-channel syringe pump (KD Scientific, USA). The system was operated at a seepage velocity of $0.8 \mathrm{~m} / \mathrm{d}$. An aqueous solution containing the four isotopologues was continuously injected from the two lowermost ports at the inlet of the flowthrough chamber. After establishing a steady-state plume (i.e., exchanging at least two pore volumes), samples were taken at the outlet ports and analyzed for the concentrations of deuterated and non-deuterated compounds.

Modeling Approach. The governing equations for contaminant transport in the two experimental setups are the 1-D Fick's second law of diffusion ${ }^{38}$ in the gel dissection tubes, and the 2-D steady state advection dispersion equation in the 2-D flow-through setup. ${ }^{39-40}$ The models used to quantitatively interpret the experimental results are based, respectively, on a numerical and an analytical solution of these governing equations. The key parameters controlling transport of the different toluene and benzene isotopologues are their diffusion coefficients and, in the flow-through setup, their transverse hydrodynamic dispersion coefficients. The governing equations and their 
91 boundary conditions are summarized in the Supporting Information together with concentration maps of the steady-state plumes in the 2-D flow-through setup.

\section{Results and Discussion}

95 Two examples of benzene and toluene spatial profiles measured in the 1-D setup after 9 and 15 days 96 of diffusion are illustrated in Figure 1. The plots show typical diffusion curves with small 97 differences between deuterated and non-deuterated compounds that are difficult to appreciate in the 98 concentration plots. The differences become apparent by plotting the ratios $\mathrm{C}_{6} \mathrm{D}_{6} / \mathrm{C}_{6} \mathrm{H}_{6}$ and $99 \mathrm{C}_{7} \mathrm{D}_{8} / \mathrm{C}_{7} \mathrm{H}_{8}$. These ratios surprisingly show an opposite trend with a decreasing pattern for toluene, 100 and a reverse, increasing trend for benzene. To quantify these observations we used a simple 101 inverse power law model, ${ }^{21,41}$ which relates the diffusion coefficients of the deuterated and nondeuterated isotopologues to their molecular masses:

$$
\frac{D_{C_{n} D_{m}}}{D_{C_{n} H_{m}}}=\left(\frac{m_{C_{n} H_{m}}}{m_{C_{n} D_{m}}}\right)^{\beta}
$$



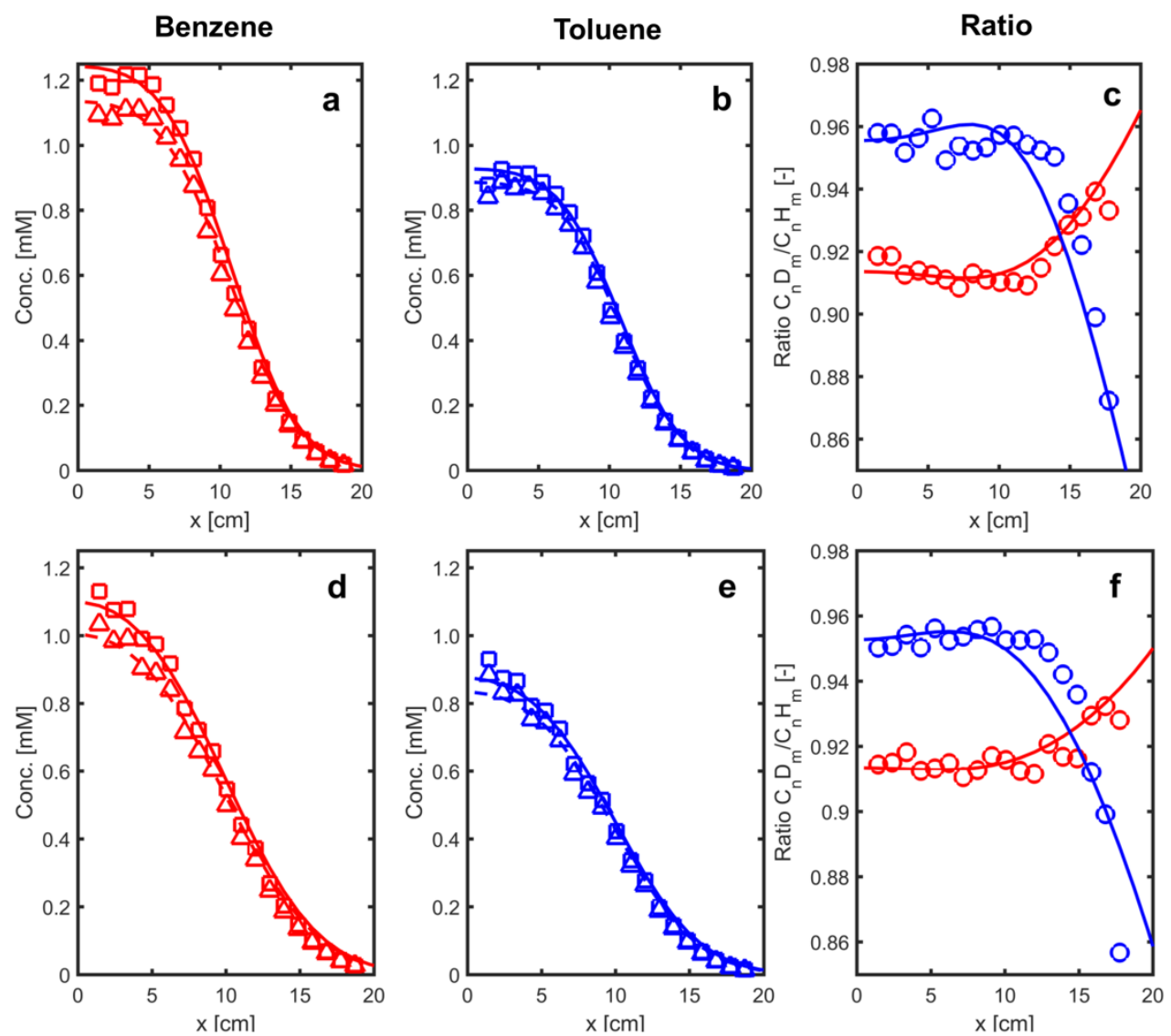

Figure 1. Spatial profiles of concentration and isotopologue ratio for benzene and toluene along the length of the diffusion tubes in the 9-day experiment $\mathrm{A}$ (panels a-c) and in the 15-day experiment $\mathrm{C}$ (panels d-f). The symbols (squares for the non-deuterated, triangles for the perdeuterated isotopologues, circles for the ratios) represent the measured data, whereas the lines are the outcomes of the simulations.

Fitting the experimental data allowed estimating the values of the diffusion coefficients for the non-

111 deuterated and D-labeled isotopologues and, thus, the value of the exponent $\beta$ expressing the mass 112 dependence of the isotopologues diffusion coefficients. The fitting procedure was carried out using 113 the function lsqnonlin implemented in MATLAB. Table 1 summarizes the results obtained for the 114 different tube experiments and reports the observed diffusive isotope fractionation. Both the 115 graphical representation of the experimental data (Figure 1) and the parameters reported in Table 1 
show a normal isotope effect for toluene and an inverse isotope effect for benzene. In the case of

117 toluene, the deuterated isotopologue has a slightly lower diffusion coefficient and results in

118 decreasing isotope ratios as the toluene species diffuse towards the pure gel medium. For benzene,

119 instead, the data show an inverse diffusive isotope effect: the molecules of the deuterated

120 isotopologue diffuse at a slightly faster rate and become enriched in the initially pure gel medium,

121 as benzene diffuses from the contamination source. This behavior was consistently observed in all experiments. The $\beta(0.444-0.490)$ values for toluene are consistent with those of previous

123 experiments, ${ }^{15}$ whereas the negative values for benzene clearly indicate an inverse isotope effect.

124 Average values characterizing normal and inverse isotope fractionation observed for toluene and

125 benzene in these diffusion experiments are: $\mathrm{D}_{\mathrm{C} 7 \mathrm{D} 8} / \mathrm{D}_{\mathrm{C} 7 \mathrm{H} 8}=0.962 \pm 0.002$ and $\mathrm{D}_{\mathrm{C} 6 \mathrm{D} 6} / \mathrm{D}_{\mathrm{C} 6 \mathrm{H} 6}=1.019 \pm 0.002$.

128 Table 1. Summary of the results for the multitracer 1-D diffusion experiments.

\begin{tabular}{cccccc}
\hline Experiment & $\begin{array}{c}\text { Time } \\
{[\text { Days }]}\end{array}$ & $\begin{array}{c}\mathbf{D}_{\mathbf{C n H m}} \\
{\left[\times 10^{-9} \mathrm{~m}^{2} \mathrm{~s}^{-1}\right]}\end{array}$ & $\begin{array}{c}\mathbf{D}_{\mathbf{C n D m}} \\
{\left[\times 10^{-9} \mathrm{~m}^{2} \mathrm{~s}^{-1}\right]}\end{array}$ & $\begin{array}{c}\boldsymbol{\beta} \\
{[-]}\end{array}$ & $\begin{array}{c}\mathbf{D}_{\mathbf{C n D m}} / \mathbf{D}_{\mathbf{C n H m}} \\
{[-]}\end{array}$ \\
\hline Benzene & & & & & \\
A & 9 & $0.958 \pm 0.040$ & $0.976 \pm 0.017$ & -0.251 & 1.019 \\
B & 9 & $0.958 \pm 0.008$ & $0.978 \pm 0.007$ & -0.279 & 1.021 \\
C & 15 & $0.956 \pm 0.021$ & $0.972 \pm 0.020$ & -0.218 & 1.016 \\
D & 15 & $0.957 \pm 0.025$ & $0.974 \pm 0.024$ & -0.238 & 1.018 \\
Toluene & & & & & \\
A & 9 & $0.800 \pm 0.010$ & $0.768 \pm 0.009$ & 0.490 & 0.960 \\
B & 9 & $0.811 \pm 0.015$ & $0.780 \pm 0.012$ & 0.460 & 0.962 \\
C & 15 & $0.798 \pm 0.012$ & $0.769 \pm 0.010$ & 0.444 & 0.964 \\
D & 15 & $0.810 \pm 0.019$ & $0.780 \pm 0.016$ & 0.453 & 0.963 \\
\hline
\end{tabular}

130 Figure 2 shows vertical profiles of concentration and isotope ratio observed for the simultaneous

131 transport of deuterated and non-deuterated benzene and toluene in the flow-through chamber. The

132 concentration trends show a typical transverse dispersion profile for plumes continuously injected in

133 a homogeneous porous medium. The isotope ratio measured at the different ports confirms the 
normal diffusive fractionation for toluene and the inverse fractionation for benzene. In fact, the ratio

135

136 $\mathrm{C}_{7} \mathrm{D}_{8} / \mathrm{C}_{7} \mathrm{H}_{8}$ decreases from the core towards the outer fringe of the plume, whereas the ratio $\mathrm{C}_{6} \mathrm{D}_{6} / \mathrm{C}_{6} \mathrm{H}_{6}$ increases towards the plume fringe. No fitting procedure was used to evaluate the data from the flow-through experiment. Instead, the experimental results were evaluated with pure forward modeling of solute transport based on the average diffusion coefficients of the 4 compounds determined in the tube diffusion setups. The key parameter in this setup is the transverse hydrodynamic dispersion coefficients, which is compound (and isotopologue) specific and was described with the following parameterization:

$$
D_{T}=D_{P}+D_{a q}\left(\frac{P e^{2}}{P e+2+4 \delta^{2}}\right)^{0.5}
$$

where $D_{P}\left[\mathrm{~L}^{2} / \mathrm{T}\right]$ denotes the velocity-independent pore diffusion coefficient; $P e=v d / D_{a q}[-]$ is the grain Péclet number, in which $d[\mathrm{~L}]$ is the average grain size diameter and $v[\mathrm{~L} / \mathrm{T}]$ is the seepage velocity; $\delta[-]$ is the ratio between the length of a pore channel and its hydraulic radius; a value of $\delta=5.37$ was determined in previous tracer experiments performed in a range of porous media, grain sizes and seepage velocities comprising the conditions of the current flow-through setup. ${ }^{42}$ This parameterization of transverse dispersion has been tested for solute transport in similar quasi 2-D systems $^{43-45}$ and verified in pore-scale studies ${ }^{46-47}$ and fully three-dimensional flow-through experiments. ${ }^{38}$ The essential feature of Eq. 2 is that it acknowledges the importance of aqueous diffusion also in the non-linear, velocity-dependent mechanical dispersion term. The good agreement of the predictive, purely forward simulations for both measured concentrations and ratios (Figure 2) shows the capability of the model to capture the diffusive fractionation effects in the flow-through system and also the accuracy of the experimentally determined values of aqueous diffusion for the deuterated and non-deuterated tracers obtained from the 1-D tube experiments. The latter compare very well with the values of aqueous diffusion coefficients computed with classical 
empirical correlations for organic compounds (see Table S2 in the Supporting Information); this 157 also indicates that sorption of toluene and benzene was not important in our experiments. The 158 values of the transverse dispersion coefficients, determined according to Eq. 2, and used in the 159 profiles and isotope ratio maps shown in Figure 2 are: $D_{T, C 7 H 8}=1.563 \times 10^{-9}, D_{T, C 7 D 8}=1.547 \times 10^{-9}$, 160 $D_{T, C 6 H 6}=1.641 \times 10^{-9}$ and $D_{T, C 6 D 6}=1.649 \times 10^{-9} \mathrm{~m}^{2} / \mathrm{s}$.

\section{Benzene}
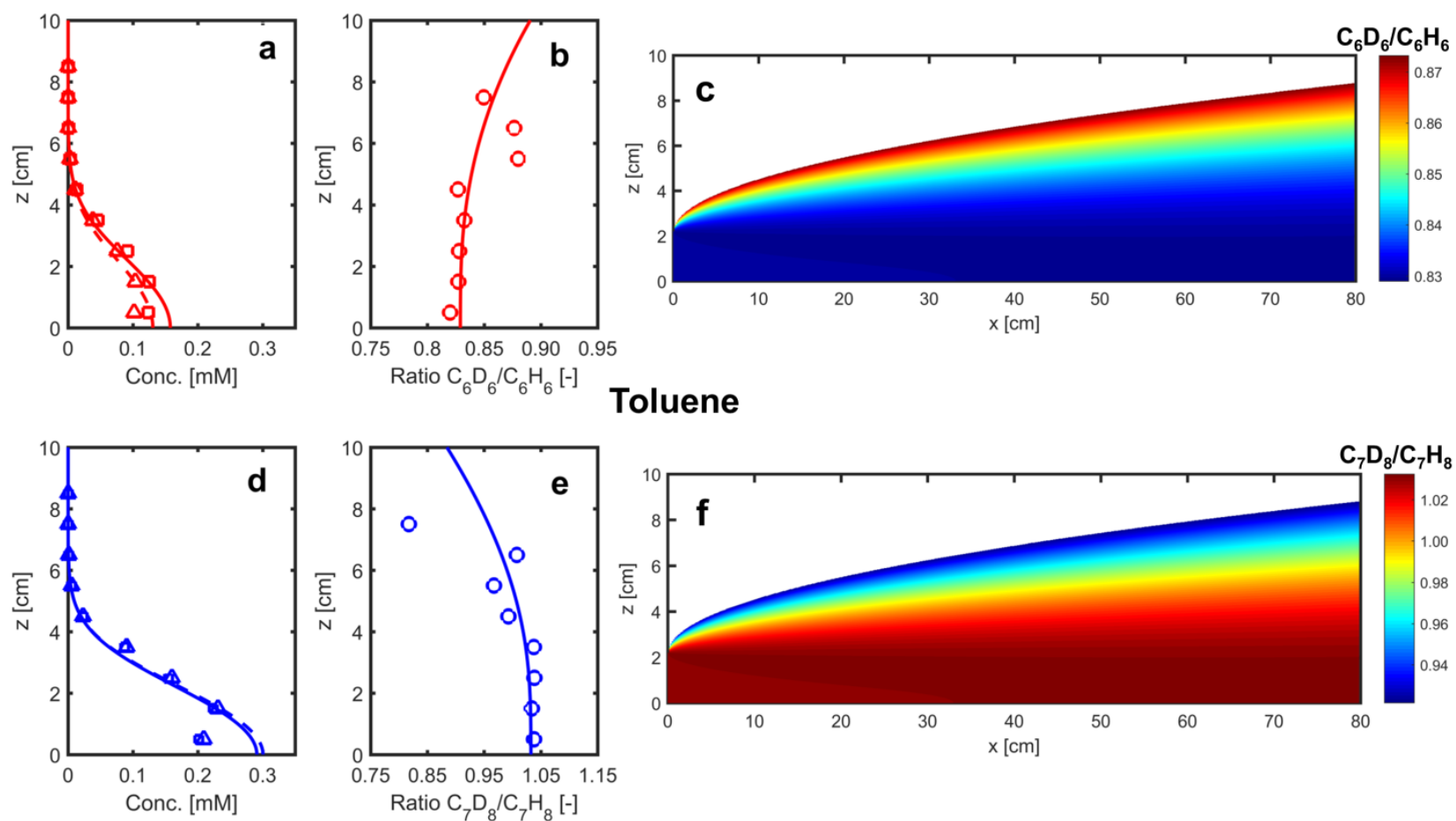

Toluene

Figure 2. Observed and simulated vertical profiles of concentrations and ratios at the outlet of the flow-through setup (a-b, d-e) and spatial maps of the isotopologue ratio for benzene (c) and toluene (f). The concentration data at the outlet ports (squares for the non-deuterated, triangles for the perdeuterated isotopologues) are average of triplicate measurements of two sampling events of the steady-state plumes, carried out after flushing 2 and 4 pore volumes (i.e., 48 and 96 hours, respectively). 
Diffusive isotope fractionation in condensed systems, such as aqueous solutions, is challenging to investigate and to conceptualize in a solid theoretical framework. Different factors that may affect diffusive isotope fractionation include solute mass, volume, shape, molecular structure, polarity, temperature and solute-solvent interactions. Mass and volume dependencies have dominated the discussion about diffusive fractionation in condensed systems and the interpretation of diffusion experiments. Models that have been proposed to capture the effects of diffusive fractionation include the so-called hydrodynamic description (e.g., Stokes-Einstein relation), as well as the extension to the condensed phase of formulations derived from Chapman-Enskog kinetic theory of gas diffusion. ${ }^{38}$ The hydrodynamic model does not include mass dependence and the key parameter is the radius and, thus, the molar volume of the solute, whereas the kinetic theory predicts an inverse square root relation with the reduced mass. ${ }^{41}$ Most of the experimental studies for different aqueous solutes have reported a mass dependence that is typically weaker than the inverse square root relation from kinetic theory. A simple power law expression (Eq. 1), in which the exponent is derived by fitting the experimental data, is the model that is most often used to relate the diffusive isotope effects to the mass of the diffusing solutes in theoretical, computational and experimental studies. $^{21,24-26,41}$ It is worth pointing out that in this simple model the exponent $\beta$ is an empirical coefficient which also lumps other factors such as steric effects and solute-solvent interactions that are likely to play an important role on diffusive isotope fractionation. Indeed, the data presented in this study show normal and inverse isotope effects that can neither be interpreted on the basis of mass dependencies nor be ascribed as purely hydrodynamic (i.e., mass independent) effects. In fact, based on the sole mass difference the deuterated benzene should have shown lower diffusion rates than the non-labeled isotopologue. Instead, an inverse effect was observed both in the tube diffusion experiments and in the flow-through setup. Our experiments also allow excluding a purely hydrodynamic interpretation of the results. Molar volumes of deuterated benzene and toluene have 
been shown to be smaller than those of the corresponding non-labeled isotopologues; for instance, Bartell and $\operatorname{Roskos}^{48}$ report the following values at $20{ }^{\circ} \mathrm{C}: V_{C 6 D 6}=88.62 \mathrm{~mL} / \mathrm{mol}, V_{C 6 H 6}=88.86$ $\mathrm{mL} / \mathrm{mol}, V_{C 7 D 8}=105.98 \mathrm{~mL} / \mathrm{mol}$, and $V_{C 7 H 8}=106.28 \mathrm{~mL} / \mathrm{mol}$. These differences are commonly attributed to the smaller effective radius of the C-D bonds compared to the C-H bonds. ${ }^{48-49}$ However, a pure hydrodynamic interpretation, based on a simple Stokes-Einstein relation and considering the molar volumes reported above, would predict very weak inverse isotope effects $\left(\mathrm{D}_{\mathrm{CnDm}} / \mathrm{D}_{\mathrm{CnHm}}=1.0009\right)$ for both compounds. This was not observed in this study, in which stronger inverse and normal diffusive isotope fractionation effects were observed for benzene and toluene, respectively. We hypothesize that the geometry of the hydration shells surrounding the aromatic molecules and the solvation dynamics play a major role for diffusive isotope fractionation of deuterated and non-deuterated benzene and toluene in aqueous solutions. For instance, a recent computational study of benzene hydration ${ }^{50}$ reports that the hydration shell of a benzene molecule has an average number of 31 water molecules and this number can change and diminish substantially upon increase of temperature and decrease of density. To explain the unexpected inverse fractionation observed for benzene, one could hypothesize a similar effect on benzene hydration due to the deuterium substitutions. Processes connected to the hydrophobic effect of aromatic molecules in solution and associated changes of density and orientation of water molecules surrounding the solute, ${ }^{51}$ as well as the structure and patterns of solute hydration ${ }^{52}$ appear to be more important than the effects due to mass and molar volume differences. This hypothesis will require future substantiation that might be provided by molecular dynamic simulations of aqueous diffusion of deuterated and non-deuterated species. Such simulations have been performed for instance for charged species ${ }^{22,53-54}$ and have contributed to elucidate the importance of the hydration shell and of the interaction between solute and water molecules for the observed isotope fractionation of different ions in aqueous solution. The different interaction of deuterated and non- 
deuterated benzene and toluene molecules with water molecules appears to be an important factor to explain the experimental observations of this study. This can be deduced also by comparing the results of this study with earlier experiments. For instance, benzene self-diffusion ${ }^{55}$ and tracer diffusion in chlorobenzene ${ }^{56}$ showed only very minor or no isotope effects during diffusion of $\mathrm{C}_{6} \mathrm{H}_{6}$ and $\mathrm{C}_{6} \mathrm{D}_{6}$ in these organic liquids, which contrasts with the rather large fractionation consistently observed in the different aqueous systems considered in our study.

We think that the normal and inverse diffusive fractionation effects observed for deuterated and non-deuterated BTEX will stimulate further research to develop a mechanistic understanding of transport and transformation of these contaminants and their labeled mixtures in different environments. Despite fractionation of $\mathrm{D} / \mathrm{H}$ substituted aromatic compounds due to biodegradation can be large and may be dominant for many transformation pathways, ${ }^{57}$ our study shows that diffusive fractionation is also significant and should be considered when these chemicals are used as tracers and diagnostic tools in environmental systems in which transport and transformation processes are typically coupled. On a broader perspective, the results of this study, showing 231 different diffusive isotope fractionation of very similar compounds, also have implications for the 232 fast developing field of compound specific stable isotope analysis (CSIA) techniques ${ }^{58-59}$, which 233 will greatly benefit from data for different environmental contaminants. The path towards 234 developing a comprehensive database of organic pollutant diffusive isotope fractionation and a 235 sound theory capturing the key mechanisms causing diffusive fractionation is inherently complex 236 but rich of intriguing future challenges.

\section{Supporting Information}

239 Description of the flow-through system and of the modeling approach used to quantitatively 240 interpret the results in the different experimental setups. 
The authors declare no competing financial interests.

\section{Acknowledgments}

The authors acknowledge the support of the Deutsche Forschungsgemeinschaft (Grant RO 4169/2-1) and the help of Chrysanthi-Elisabeth Nika, Hanne Bøggild and Mikael Emil Olsson for assistance in

the experimental work.

\section{References}

1. Wiedemeier, T. H.; Rifai, H. S.; Newell, C. H.; Wilson, T. H., Natural attenuation of fuels and chlorinated solvents in the subsurface. John Wiley, New York, 1999, 617 p.

2. Thierrin, J.; Davis, G. B.; Barber, C., A Groundwater Tracer Test with Deuterated Compounds for Monitoring In-Situ Biodegradation and Retardation of Aromatic Hydrocarbons. Ground Water 1995, 33, 469-475.

3. Reusser, D. E.; Istok, J. D.; Beller, H. R.; Field, J. A., In situ transformation of deuterated toluene and xylene to benzylsuccinic acid analogues in BTEX-contaminated aquifers. Environ. Sci. Technol. 2002, $36,4127-4134$.

4. Fisher, A.; Bauer, J.; Meckenstock, R. U.; Stichler, W.; Griebler, C.; Maloszewski, P.; Kastner, M.; Richnow, H. H., A multitracer test proving the reliability of Rayleigh equation-based approach for assessing biodegradation in a BTEX contaminated aquifer. Environ. Sci. Technol. 2006, 40, 4245-4252.

5. Gieg, L. M.; Alumbaugh, R. E.; Field, J.; Jones, J.; Istok, J. D.; Suflita, J. M., Assessing in situ rates of anaerobic hydrocarbon bioremediation. Microb. Biotechnol. 2009, 2, 222-233.

6. Cozzarelli, I. M.; Bekins, B. A.; Eganhouse, R. P.; Warren, E.; Essaid, H. I., In situ measurements of volatile aromatic hydrocarbon biodegradation rates in groundwater. J. Contam. Hydrol. 2010, 111, 48-64.

7. Parker, B. L.; Chapman, S. W.; Guilbeault, M. A. Plume persistence caused by back diffusion from thin clay layers in a sand aquifer following TCE source-zone hydraulic isolation. J. Contam. Hydrol. 2008, 102, 86-104.

8. Carrera, J.; Sanchez-Vila, X.; Benet, I.; Medina, A.; Galarza, G.; Guimera, J., On matrix diffusion: formulations, solution methods and qualitative effects. Hydrogeol. J. 1998, 6,178-90.

9. Rolle, M.; Chiogna, G.; Hochstetler, D. L.; Kitanidis, P. K., On the importance of diffusion and compound-specific mixing for groundwater transport: An investigation from pore to field scale, $J$. Contam. Hydrol. 2013,153, 51-68.

10. Rolle, M.; Kitanidis, P.K., Effects of compound-specific dilution on transient transport and solute breakthrough: A pore-scale analysis. Adv. Water Resour. 2014, 71, 186-199.

11. LaBolle, E. M.; Fogg, G. E.; Eweis, J. B.; Gravner, J.; Leaist, D. G., Isotopic fractionaB.;tion by diffusion in groundwater. Water Resour. Res. 2008, 44.

12. Rolle, M.; Chiogna, G.; Bauer, R.; Griebler, C.; Grathwohl, P., Isotopic Fractionation by Transverse Dispersion: Flow-through Microcosms and Reactive Transport Modeling Study. Environ. Sci. Technol. 2010, 44, 6167-6173.

13. Eckert, D.; Rolle, M.; Cirpka, O. A., Numerical simulation of isotope fractionation in steady-state bioreactive transport controlled by transverse mixing. J. Contam. Hydrol. 2012, 140, 95-106.

14. Van Breukelen, B. M.; Rolle, M., Transverse Hydrodynamic Dispersion Effects on Isotope Signals in Groundwater Chlorinated Solvents' Plumes. Environ. Sci. Technol. 2012, 46, 7700-7708. 
15. Jin, B.; Rolle, M.; Li, T.; Haderlein, S.B., Diffusive fractionation of BTEX and chlorinated ethenes in aqueous solution: quantification of spatial isotope gradients. Environ. Sci. Technol. 2014, 48, 6141-6150.

16. Wanner, P.; Hunkeler, D., Carbon and chlorine isotopologue fractionation of chlorinated hydrocarbons during diffusion in water and low permeability sediments. Geochim. et Cosmochim. Acta 2015, 157, 198-212.

17. Jähne, B.; Heinz, G.; Dietrich, W., Measurement of the diffusion coefficients of sparingly soluble gases in water. Jounal of Geophysical Research: Oceans 1987, 92, 10767-10776.

18. Eggenkamp, H. G. M.; Middelburg, J. J.; Kreulen, R., Preferential diffusion of $35 \mathrm{Cl}$ relative to $37 \mathrm{Cl}$ in sediments of Kau Bay, Halmahera, Indonesia. Chem. Geol. 1994, 116, 317-325.

19. Prinzhofer, A.; Pernaton, E.; Isotopically light methane in natural gas: bacterial imprint or diffusive fractionation? Chem. Geol. 1997, 142, 193-200.

20. Schloemer, S.; Krooss, B. M., Molecular transport of methane, ethane and nitrogen and the influence of diffusion on the chemical and isotopic composition of natural gas accumulations. Geofluids 2004, 4, 81108.

21. Richter, F. M.; Mendybaev, R. A.; Christensen, J. N.; Hutcheon, I. D.; Williams, R. W.; Sturchio, N. C.; Beloso, A. D., Kinetic isotopic fractionation during diffusion of ionic species in water. Geochim. Cosmochim. Acta 2006, 70, 277-289.

22. Bourg, I. C.; Sposito, G., Molecular dynamics simulations of kinetic isotope fractionation during the diffusion of ionic species in liquid water. Geochim. Cosmochim. Acta 2007, 71, 5583-5589.

23. Donahue, M. A.; Werne, J. P.; Meile, C.; Lyons, T. W., Modeling sulfur isotope fractionation and differential diffusion during sulfate reduction in sediments of the Cariaco Basin. Geochim. Cosmochim. Acta 2008, 72, 2287-2297.

24. Bourg, I. C.; Sposito, G., Isotopic fractionation of noble gases by diffusion in liquid water: Molecular dynamics simulations and hydrologic applications. Geochim. Cosmochim. Acta 2008, 72, 2237-2247.

25. Eggenkamp, H. G. M.; Coleman, M. L., The effect of aqueous diffusion on the fractionation of chlorine and bromine stable isotopes. Geochim. Cosmochim. Acta 2009, 73, 3539-3548.

26. Bourg, I. C.; Richter, F. M.; Christensen, J. N.; Sposito, G., Isotopic mass dependence of metal cation diffusion coefficients in liquid water. Geochim. Cosmochim. Acta 2010, 74, 2249-2256.

27. Beekman, H. E.; Eggenkamp, H. G. M.; Appelo, C. A. J., An integrated modelling approach to reconstruct complex solute transport mechanisms $-\mathrm{Cl}$ and delta $\mathrm{Cl}-37$ in pore water of sediments from a former brackish lagoon in The Netherlands. Appl. Geochem. 2011, 26, 257-268.

28. Wortmann, U. G.; Chrnyavsky, B. M., The significance of isotope specific diffusion coefficients for reaction-transport models of sulfate reduction in marine sediments. Geochim. Cosmochim. Acta 2011, 75, 3046-3056.

29. Tempest, K.; Emerson, S., Kinetic isotope fractionation of argon and neon in during air-water transfer. Marin. Chem. 2013,153, 39-47.

30. Richter, F. M.; Liang, Y.; Davis, A. M.; Isotope fractionation by diffusion in molten oxides. Geochim. Cosmochim. Acta 1999, 63, 2853-2861.

31. Richter, F. M.; Davis, A. M.; DePaolo, D. J.; Watson, E. B.; Isotope fractionation by chemical diffusion between molten basalt and rhyolite. Geochim. Cosmochim. Acta 2003, 67, 3905-3923.

32. Watkins, M. W.; DePaolo, D. J.; Ryerson, F. J.; Peterson, B. T., Influence of liquid structure on diffusive isotope separation in molten silicates and aqueous solutions. Geochim. Cosmochim. Acta 2011, 75, 31033118.

33. Watkins, M. W.; Liang, Y.; Richter, F. M.; Ryerson, F. J.; DePaolo, D. J., Diffusion of multi-isotopic chemical species in molten silicates. Geochim. Cosmochim. Acta 2014, 139, 313-326. 
34. Mills, R., Search for isotope effects in the self-difusion of benzene and cyclohexane at $25^{\circ}$. J. Phys. Chem. 1975, 79, 852-853.

35. Mills, R.; Harris, K. R., The effect of isotopic substitution on diffusion in liquids. Chem. Soc. Rev. 1976, 5, 215-231.

36. Freer, R.; Sherwood, J. N., Diffusion in organic liquids. J.C.S. Faraday I 1980, 76, 1030-1037.

37. Haberer, C. M.; Rolle, M.; Cirpka, O.A.; Grathwohl P., Oxygen transfer in a fluctuating capillary fringe. Vadose Zone J. 2012, 11.

38. Cussler, E.L.; Diffusion - mass transfer in fluid systems. Cambridge University Press ( $3^{\text {rd }}$ Edition), 2009, $631 \mathrm{p}$.

39. Cirpka, O.A.; Rolle, M.; Chiogna, G.; de Barros, F.P.J.; Nowak, W, Stochastic evaluation of mixingcontrolled steady-state plume lengths in two-dimensional heterogeneous domains. J. Contam.Hydrol. 2012, 138-139, 22-39.

40. Muniruzzaman, M.; Haberer, C.M.; Grathwohl, P.; Rolle, M., Multicomponent ionic dispersion during transport of electrolytes in heterogeneous porous media: Experiments and model-based interpretation. Geochim. Cosmochim. Acta 2014, 656-669.

41. Bhattacharyya, S.; Bagchi, B., Power law mass dependence of diffusion: A mode coupling theory analysis. Phys. Rev. E 2000, 61, 3850-3856.

42. Ye, Y.; Chiogna, G.; Cirpka, O. A.; Grathwohl, P.; Rolle M., Experimental investigation of compoundspecific dilution of solute plumes in saturated porous media: 2-D vs. 3-D flow-through systems. $J$. Contam. Hydrol. 2015, 172, 33-47.

43. Chiogna, G.; Eberhardt, C.; Grathwohl, P; Cirpka, O. A.; Rolle, M., Evidence of compound-dependent hydrodynamic and mechanical transverse dispersion by multitracer laboratory experiments. Environ. Sci. Technol.2010, 44, 688-693.

44. Rolle, M.; Muniruzzaman, M.; Haberer, C. M.; Grathwohl, P., Coulombic effects in advectiondominated transport of eletrolytes in porous media: Multicomponent ionic dispersion. Geochim. Cosmochim. Acta 2013, 120, 195-205.

45. Muniruzzaman, M.; Rolle, M., Impact of multicomponent ionic transport on $\mathrm{pH}$ fronts propagation in saturated porous media. Water Resour. Res. 2015, 51, 6739-6755.

46. Rolle, M.; Hochstetler, D. L.; Chiogna, G.; Kitanidis, P. K.; Grathwohl P., Experimental investigation and pore-scale modeling interpretation of compound-specific transverse dispersion in porous media. Transp. Porous Med. 2012, 93, 347-362.

47. Hochstetler, D. L.; Rolle, M.; Chiogna, G.; Haberer, C. M.; Grathwohl, P.; Kitanidis, P. K., Effects of compound-specific transverse mixing on steady-state reactive plumes: Insights from pore-scale simulations and Darcy-scale experiments. Adv. Water Resour. 2013, 54, 1-10.

48. Bartell, L. S.; Roskos, R. R.; Isotope effects on molar volume and surface tension: simple theoretical model and experimental data for hydrocarbons. J. Phys. Chem. 1966, 44, 457-463.

49. Dunitz, J.; Ibberson, R. M., Is deuterium always smaller than protium? Angewandte ChemieInternational Edition 2008, 120, 4276-4278.

50. Choudhary, A.; Chandra, A., Spatial and orientational structure of the hydration shell of benzene in suband supercritical water. J. Phys. Chem. B 2015, 119, 8600-8612.

51. Raschke, T.; Levitt, M., Nonpolar solutes enhance water structure within hydration shells while reducing interactions between them. PNAS 2005, 102, 6777-6782.

52. Schravendijk, P.; van der Vegt, N.F.A., From hydrophobic to hydrophilic solvation: An application to hydration of benzene. J. Chem. Theory Comput. 2005, 1, 643-652.

53. Moller, K. B.; Rey, R.; Masia, M.; Hynes, J. T., On the coupling between molecular diffusion and solvation shell exchange. J. Chem. Phys. 2005, 122. 
54. Hofmann, A. E.; Bourg, I. C.; DePaolo, D. J., Ion desolvation as a mechanism for kinetic isotope fractionation in aqueous systems. PNAS 2012, 109, 18689-18694.

55. Mills, R., Diffusion relationships in the binary system benzene-perdeuteriobenzene at 25 C. J. Phys. Chem. 1976, 80, 888-890.

56. Shankland, I. R.; Dunlop, P. J.; Barr, L. W., Isotope effect in liquids as shown by tracer diffusion at 25 C of several labeled benzenes in benzene, chlorobenzene, n-octane, and cyclohexane, and the relationship to the isotope effect in solids. Phys. Rev. B 1975, 12, 2249-2252.

57. Morasch, B; Richnow, H-H.; Schink, B.; Meckenstock, R.U., Stable hydrogen and carbon isotope fractionation during microbial toluene degradation: mechanistic and environmental aspects. Appl. Environ. Microbiol. 2001, 67, 4842-4849.

58. Thullner, M.; Centler, F.; Richnow, H. H.; Fischer, A., Quantification of organic pollutant degradation in contaminated aquifers using compound specific stable isotope analysis - Review of recent developments. Org. Geochem. 2012, 42, 1440-1460.

59. Hatzinger, P. B.; Bohlke, J.; Sturchio, N. C., Application of stable isotope ratio analysis for biodegradation monitoring in groundwater. Current opinion in biotechnology 2013, 24, 542-549. 